ARTIGO ORIGINAL ORIGINAL ARTICLE

\title{
Número necessário a tratar (NNT) e custo por evento evitado (COPE) de enzalutamida e acetato de abiraterona para o tratamento de câncer de próstata resistente à castração que falharam a terapia de privação de androgênio
}

\author{
Number needed to treat (NNT) and cost of preventing an event \\ (COPE) of enzalutamide vs. abiraterone acetate plus prednisone for \\ the treatment of metastatic castration-resistant prostate cancer \\ ( $m C R P C)$ patients who failed androgen deprivation therapy (ADT)
}

Stephen Stefani' ${ }^{1}$, André Sasse ${ }^{2}$, André Fay ${ }^{3}$, Hellen Kim ${ }^{4}$, Marcia Abadi ${ }^{5}$,

Vanessa Teich', Gilberto Lopes ${ }^{7}$

DOI: $10.21115 /$ JBES.v8.n2.p141-148

\section{Palavras-chave:}

enzalutamida, abiraterona, neoplasias de próstata resistentes à castração, número necessário a tratar

\section{RESUMO}

Objetivo: O objetivo deste estudo foi estimar o número necessário a tratar (NNT) e custo por evento evitado (COPE) de enzalutamida (ENZ) em comparação com abiraterona+prednisona (AA+P) em 12 e 24 meses sob perspectiva do sistema de saúde suplementar em pacientes com câncer de próstata resistente à castração metastático (CPRCM) sem quimioterapia prévia. Métodos: $O$ NNT é calculado pelo inverso da diferença do risco absoluto de uma intervenção versus placebo; adicionalmente, o COPE representa o NNT multiplicado pelo custo de tratamento total de um período determinado. O risco absoluto de ENZ e AA+P e seus respectivos controles foram obtidos das curvas de sobrevida livre de progressão radiográfica (SLPr) e sobrevida global (SG) dos estudos PREVAIL e COU-AA-302, respectivamente. A duração de tratamento média no horizonte de 24 meses foi estimada utilizando a área sob a curva das respectivas curvas de SLPr. Os resultados foram a comparação entre ENZ e $\mathrm{AA}+\mathrm{P}$ versus seus respectivos placebos em 12 e 24 meses para NNT e COPE. O custo total de tratamento consistiu em custos de medicamento, monitoramento, e manejo de eventos adversos ( $\geq 1 \%$, eventos de interesses especiais). Resultados: A análise de 12 meses resultou em NNTSG/ENZ=12,79; NNTSLPr/ENZ= 2,59; NNTSG/AA+P= 116,28; NNTSLPr/AA+P= 4,72 e COPESG/ENZ= BRL 1.626.583; COPESLPr/ENZ = BRL 329.701; COPESG/AA+P=BRL 15.144.886; COPESLPr/AA+P=BRL 614.368. Para a análise de 24 meses, os resultados foram: NNTSG/ENZ= 11,00; NNTSLPr/ENZ=3,58; NNTSG/AA+P= 16,56; NNTSLPr/AA+P=5,00 e COPESG/ENZ= BRL 1.965.454; COPESLPr/ENZ= BRL 639.327; COPESG/ $A A+P=B R L$ 2.833.580; COPESLPr/AA+P= BRL 855.741. Conclusão: Para ambos horizontes de tempo, os resultados foram favoráveis para ENZ vs. AA+P em pacientes com CPRCM.
Recebido em: 30/06/2016. A provado para publicação em: 10/08/2016.

1. Médico Oncologista, Instituto do Câncer Mãe de Deus, Rio Grande do Sul, Brasil. Professor, Fundação Unimed, Porto Alegre, Brasil. 2. Médico Oncologista, Centro de Evidência em Oncologia (CEVON) da Universidade Estadual de Campinas (UNICAMP), São Paulo, Brasil. 3. Médico Oncologista, Instituto do Câncer Mãe de Deus, Rio Grande do Sul, Brasil. Professor, Faculdade de Medicina da Pontifícia Universidade Católica do Rio Grande do Sul (PUCRS), Rio Grande do Sul, Brasil.

4. Analista de projetos, Sense Company, São Paulo, Brasil.

5. Gerente médico senior América Latina, Astellas Farma, Inc., São Paulo, Brasil.

6. Vice-presidente, Sense Company, São Paulo, Brasil.

7. Chief Medical and Scientific Officer, Grupo Oncoclínicas do Brasil, São Paulo, Brasil/Professor assistente, Universidade Johns Hopkins. Instituição onde o trabalho foi executado: Sense Company, São Paulo, Brasil.

Autor Correspondente: Vanessa Teich, Rua São Paulino, 110 - Vila Mariana - São Paulo/SP, (11) 4081-1913, vanessa.teich@wearesense.company.

Declaração: Enzalutamida é um princípio ativo co-desenvolvido pela Astellas Pharma, Inc., Northbrook, IL, EUA e pela Medivation, Inc., San Francisco, CA, EUA. Esta pesquisa e a assistência editorial no desenvolvimento deste manuscrito foram feitos pelos autores em colaboração com a Sense Company e foram financiados pela Astellas Brasil.

Informações sobre auxílios recebidos sob a forma de financiamento, equipamentos ou medicamentos: Este estudo foi financiado pela Astellas Farma Brasil. Não obstante, convém salientar que não houve qualquer interferência ou ingerência da referida empresa junto aos autores para a alteração de qualquer resultado obtido pelos autores. 


\section{Keywords:}

enzalutamide, abiraterone, prostatic neoplasms, number needed to treat

\begin{abstract}
Objective: The aim of this study was to estimate the NNT and COPE of enzalutamide (ENZ) in comparison with abiraterone acetate+prednisone (AA+P) over a 12-month and 24-month period from the Supplementary Health System perspective in metastatic castration-resistant prostate cancer patients who are chemotherapy naïve (MCRPC). Methods: The NNT is calculated by the inverse of the absolute risk reduction of an intervention vs. control; additionally, COPE represents the NNT multiplied by total cost of treatment in a pre-defined period. The absolute risk of ENZ and AA+P, and their respective control treatments, were obtained from the Kaplan Meier curves for the co-primary end points of radiographic progression free survival (rPFS) and overall survival (OS) from the clinical studies PREVAIL and COU-AA-302, respectively. Mean treatment duration was estimated utilizing the area under curve (AUC) technique from the respective intervention rPFS curves. The results analyzed ENZ or AA+P versus its respective placebo at 12 and 24 months for NNT and COPE. Total treatment cost consisted of drug cost, monitoring cost and adverse event ( $>=1 \%$ incidence and special interest adverse events) related cost. Results: The 12 month analysis resulted in NNTOS/ENZ=12.79; NNTr$\mathrm{PFS} / \mathrm{ENZ}=2.59 ; \mathrm{NNTOS} / \mathrm{AA}+\mathrm{P}=116.28 ; \mathrm{NNTrPFS} / \mathrm{AA}+\mathrm{P}=4.72$ and $\mathrm{COPEOS} / \mathrm{ENZ}=\mathrm{BRL}$ 1,626,583; COPErPFS/ENZ= BRL 329,701; COPEOS/AA+P=BRL 15,144,886; COPErPFS/AA+P=BRL 614,368. For the 24-month analysis, the results were: NNTOS/ENZ=11.00; NNTrPFS/ENZ= 3.58; NNTOS/AA+P=16.56; NNTrPFS/AA+P = 5.00 and COPEOS/ENZ= BRL 1,965,454; COPErPFS/ENZ= BRL 639,327; COPEOS/ $A A+P=B R L$ 2,833,580; COPErPFS/AA+P = BRL 855,741. Conclusion: Across the 12- and 24-month time horizons, the NNT and COPE was favorable for ENZ vs. AA+P in patients with MCRPC.
\end{abstract}

\section{Introdução}

O câncer de próstata apresenta alta incidência mundial, sendo a neoplasia mais incidente entre os homens. Ao ser diagnosticado em estádios iniciais da doença, pacientes com câncer de próstata apresentam alta taxa de sobrevida em 5 anos (National Cancer Institute, 2015), entretanto, essa doença ainda se posiciona como a quinta maior causa de morte por câncer na população masculina mundial (World Health Organization, 2012).

No Brasil, 61.200 novos casos de câncer de próstata estão estimados para o ano de 2016 (Ministério da Saúde, Brasil, Instituto Nacional do Câncer José Alencar Gomes da Silva, 2015). De acordo com dados do Instituto Nacional do Câncer José Alencar Gomes da Silva (INCA), foi obtida uma média de 812.644 anos potenciais de vida perdidos por câncer de próstata durante o período de 2000 a 2013 (Ministério da Saúde, Brasil, Instituto Nacional do Câncer José Alencar Gomes da Silva, 2014), demonstrando o impacto social e econômico promovido por esse câncer na sociedade brasileira.

Ao ser diagnosticado, o tratamento de câncer de próstata é iniciado com a terapia de privação de androgênio (TPA) (Recine \& Sternberg, 2015). De maneira geral, os pacientes que recorreram após terapia local ou apresentam disseminação à distância, respondem ao tratamento padrão. Porém, os pacientes que apresentam progressão de doença em vigência de TPA avançam para o que é denominado de câncer de próstata resistente à castração metastático (CPRCM). O CPRCM pode apresentar um dos ou a combinação dos seguintes sinais: contínua elevação dos níveis séricos de antígeno específico da próstata (PSA), progressão da doença pré-existente ou o surgimento de novas metástases (Hotte \& Saad, 2010).
Considerando as diferentes opções terapêuticas disponíveis para o tratamento de CPRCM, diretrizes nacionais e internacionais sugerem a utilização de novos agentes hormonais, dentre elas o acetato de abiraterona e a enzalutamida, baseada no importante papel da via de sinalização do receptor de androgênio que permanece neste cenário (Beneficência Portuguesa de São Paulo \& Sociedade Beneficente Israelita Brasileira, 2016; National Comprehensive Cancer Network, 2016; Parker et al., 2015). Ambos apresentam indicações tanto para pacientes com CPRCM assintomáticos e levemente sintomáticos, após falha da terapia de privação androgênica, quanto para aqueles que receberam quimioterapia prévia com docetaxel (Astellas Farma Brasil, 2016; Janssen-Cilag Farmacêutica Ltda., 2016).

No caso da indicação para pacientes CPRCM que não receberam quimioterapia prévia, estudos clínicos de fase III comprovaram a eficácia de ambos os medicamentos. A enzalutamida, por meio do estudo PREVAIL, demonstrou meIhores perfis de sobrevida livre de progressão radiográfica $(H R=0,19 ;$ IC95\%: 0,15 a 0,23; $p<0,001)$ e sobrevida global ( $H R=$ 0,71; IC95\%: 0,60 a 0,84; $p<0,001$ ) quando comparada ao placebo, promovendo uma redução de $29 \%$ do risco de morte ao utilizar o medicamento (Beer et al., 2015, 2014).

O acetato de abiraterona associado à prednisona também demonstrou benefícios clínicos ao ser comparado à utilização de placebo associado à prednisona. A partir do estudo COU-AA-302, a abiraterona associada à prednisona apresentou sobrevida livre de progressão radiográfica ( $H R=0,53 ;$ IC95\%: 0,45 a 0,62; $p<0,001)$ e sobrevida global ( $H R=0,81$; IC95\%: 0,70-0,93; $p=0,0033$ ) estatisticamente favoráveis (Ryan et al., 2013, 2015).

Em 2015, um estudo realizou uma análise econômica envolvendo a enzalutamida versus abiraterona+prednisona 
(AA+P) em homens com CPRCM que receberam quimioterapia prévia (Stefani et al., 2015), demonstrando o benefício da enzalutamida em número necessário a tratar (NNT) e custo por evento evitado, em inglês, Cost of Preventing an Event (COPE). O COPE é uma possível alternativa para avaliar um medicamento, cujos resultados de estudos controlados randomizados estão disponíveis de uma maneira mais simplificada e acessível, na ausência de uma avaliação de custo-efetividade ou comparação direta de dados de eficácia (Maharaj, 2008).

Considerando a indicação de tratamento para pacientes com CPRCM que não receberam terapia prévia com docetaxel, o atual estudo tem o objetivo de avaliar o NNT e o COPE da enzalutamida versus o AA+P em pacientes com CPRCM pós-ADT sob a perspectiva do sistema de saúde suplementar.

\section{Métodos}

O cálculo do custo de tratamento dos medicamentos foi realizado utilizando as posologias indicadas nas bulas brasileiras registradas de enzalutamida e abiraterona. No caso de acetato de abiraterona, a dosagem de prednisona foi adicionada ao cálculo em 100\% dos pacientes, uma vez que sua administração deve ser realizada em combinação com prednisona ou prednisolona. Já para a enzalutamida, a utilização de prednisona é opcional, sendo a porcentagem utilizada para essa população (4\%) obtida do estudo pivotal PREVAlL (Astellas Farma Brasil, 2016; Beer et al., 2014; Janssen-Cilag Farmacêutica Ltda., 2016). A análise foi realizada considerando dois horizontes de tempo: 12 e 24 meses de tratamento.

Sob a perspectiva do sistema de saúde suplementar, os preços dos medicamentos foram obtidos pela lista oficial publicada pela Câmara de Regulação do Mercado de Medicamentos (CMED), considerando o preço fábrica com 18\% de ICMS (Ministério da Saúde, Brasil, Agência Nacional Vigilância Sanitária, 2016).

Os custos relacionados ao manejo de eventos adversos (EAs) e acompanhamento do paciente foram incluídos no resultado de custos totais. Essas informações foram coletadas por meio de um micro-custeio, baseado na opinião de especialistas, utilizando a tabela de Classificação Brasileira Hierarquizada de Procedimentos Médicos (CBHPM) (Associação Médica Brasileira, 2015).

A taxa de cada EA foi obtida por meio de informações dos estudos pivotais (PREVAIL e COU-AA-302) (Beer et al., 2014; Ryan et al., 2015), sendo incluídos apenas os EAs graus 3 e 4 cuja taxa fosse $\geq 1 \%$, e os casos de EAs de interesses especiais descritos na bulas americanas fornecidas pela Food and Drug Administration (FDA) (U.S. Food and Drug Administration, 2016a, 2016b). Esses EAs foram considerados, pois os custos referentes ao manejo desses eventos, especificamente, são os mais significantes devido à necessidade de intervenção médica e hospitalização de acordo com sua gravidade (Ministério da Saúde, Brasil, Agência Nacional Vigilância Sanitária, 2015).

Além disso, houve a adição dos custos de monitoramento de pacientes durante o período de tratamento. Para os pacientes tratados com abiraterona, a bula preconiza a necessidade de acompanhar os níveis séricos de transaminases e bilirrubina a cada duas semanas durante os três primeiros meses de tratamento, e depois disso, mensalmente (Janssen-Cilag Farmacêutica Ltda., 2016). Como o tratamento com enzalutamida não possui essa necessidade, apenas os custos referentes às visitas médicas mensais para acompanhamento foram adicionados aos custos de monitoramento (Equação 1).

Equação 1. Cálculo de custo de tratamento

Custo de tratamento $=$ Custo do medicamento + Custo de manejo de EAs + Custo de acompanhamento

O NNT é a medida utilizada para medir o impacto de uma terapia, estimando o número necessário de pacientes tratados a fim de impactar um indivíduo (Stefani et al., 2015). O COPE consiste em um cálculo envolvendo o NNT e o custo anual de tratamento (Maharaj, 2008), representando o custo real para cada paciente em que há sucesso com o tratamento, evitando a ocorrência de um evento indesejado (Stefani et al., 2015).

O cálculo do NNT é o inverso da redução de risco absoluto de um evento e, para executá-lo, foi necessária a obtenção das taxas de sucesso da terapia de interesse e de seu comparador (Equação 2). Neste caso, as informações clínicas foram os desfechos primários dos estudos de fase III de enzalutamida e abiraterona - sobrevida global (SG) e sobrevida livre de progressão radiográfica (SLPr). Para ambos os casos, os medicamentos foram comparados a placebo, na ausência de estudos head-to-head (comparação direta) entre enzalutamida e abiraterona.

Equação 2. Cálculo de número necessário a tratar (NNT)

NNT $=1 /$ (Taxa de Sucesso tratamento -

Taxa de Sucesso controle)

A duração de tratamento para o horizonte de tempo de 12 meses foi assumida em 12 meses, uma vez que os resultados de SLPr foram acima desse valor para ambas as opções terapêuticas em seus respectivos estudos clínicos. Já para o horizonte de tempo de 24 meses, o cálculo de área sob a curva (AUC), das curvas de SLPr de enzalutamida e abiraterona, foi realizado, assumindo que o tratamento é interrompido no momento da progressão da doença.

Apesar de envolver dois estudos distintos, os estudos pivotais COU-AA-302 e PREVAIL (Beer et al., 2014; Ryan et al., 2015) apresentam populações de pacientes com caracterís- 
ticas semelhantes e comparáveis. Em ambos os estudos, os pacientes apresentavam critérios de inclusão semelhantes, como a comprovação histológica e citológica de adenocarcinoma de próstata, com metástases, progressão de antígeno prostático específico (PSA), progressão radiográfica tanto em tecidos moles quanto em ossos, apresentação de Eastern Cooperative Oncology Group (ECOG) 0 ou 1, privação de androgênio com níveis de testosterona menores que 50 ng por dl, assintomáticos ou com sintomas médios. Porém, apesar das populações de ambos os estudos apresentarem características semelhantes, não foi possível realizar uma comparação indireta de enzalutamida e AA+P, o que se justifica pelas diferenças entre os grupos controles dos estudos PREVAIL e COU-AA-302. O estudo PREVAIL realizou a comparação entre enzalutamida versus placebo apenas, enquanto os braços do estudo COU-AA-302 consistiam em acetato de abiraterona ou placebo associados à prednisona, exemplificando a inconsistência dos grupos com placebo. Por esse motivo, optou-se por realizar o estudo comparando as opções terapêuticas (enzalutamida e AA+P) aos seus respectivos grupos controle.

O COPE é o produto do custo de tratamento e o NNT, e esse cálculo foi realizado tanto para o tratamento com enzalutamida quanto para a abiraterona (Equação 3).

Equação 3. Cálculo do custo por evento evitado (COPE)

COPE $=$ custo de tratamento em um

período determinado * NNT

O intervalo de confiança de 95\% (IC95\%) foi calculado para o NNT, sendo possível calcular o mesmo para o COPE (Equação 4).

Equação 4. Cálculo do IC95\% de NNT

Redução do Risco Absoluto (RRA) = Taxa de

RespostaTratamento - Taxa de RespostaControle

Erro Padrão (RRA) $=\sqrt{ }[$ Taxa de RespostaTratamento2 $*(1-$

Taxa de RespostaTratamento)/nTratamento+ Taxa de

RespostaControle2*(1- Taxa de RespostaControle)/ncontrole] IC95\% (RRA) $=$ RRA $\pm[1,96 *$ Erro Padrão (RRA) $]$

IC95\% (NNT) $=1 /$ IC95\%(RRA)

$\mathrm{n}=$ número de pacientes em risco

\section{Resultados}

Os preços dos medicamentos obtidos pela lista atualizada da CMED, assim como a quantidade utilizada e custo unitário estão exemplificados na Tabela 1. É importante ressaltar que o custo de tratamento com enzalutamida foi superior ao custo de tratamento com abiraterona em 24 meses, pois os pacientes tratados com enzalutamida permanecem por mais tempo em tratamento quando comparados a aqueles tratados com abiraterona associada à prednisona (16,9 vs. 15,8 meses, respectivamente). Já em 12 meses, ambos os tratamentos tiveram a mesma duração.

As frequências de administração e quantidades utilizadas foram obtidas pelas bulas de enzalutamida e abiraterona (Astellas Farma Brasil, 2016; Janssen-Cilag Farmacêutica Ltda., 2016), assim como está descrito na Tabela 2.

Os resultados de EAs foram os mesmos tanto para o horizonte de tempo de 12 meses quanto para os 24 meses (Tabela 3).

\section{Horizonte de tempo 12 meses}

Os dados de eficácia foram obtidos pelos estudos clínicos, e o corte da curva no tempo de 12 meses resultou em SG de enzalutamida 0,92 versus 0,84 do placebo, e SLPr de 0,66 em comparação a 0,28 do placebo. Já a combinação de abiraterona e prednisona resultou em porcentagem de pacientes vivos de 0,91 em comparação a 0,90 de placebo mais prednisona. A SLPr foi de 0,58 versus 0,37 (Tabela 4). Esses dados possibilitaram o cálculo de NNT para ambas as opções terapêuticas, sendo exemplificados na Tabela 5. Para SG, NNT de enzalutamida foi de 12,79, enquanto AA+P resultou em 116,28. Já para a SLPr, enzalutamida obteve um NNT de 2,59 e AA+P de 4,72.

Para o horizonte de tempo de 12 meses, a duração de tratamento foi assumida em 12 meses, uma vez que os dados clínicos sugerem que até esse ponto da curva não haveria a progressão da doença. O custo de acompanhamento de AA+P consistiu na soma dos custos envolvendo os exames e visitas médicas durante o período de 12 meses, resultando em R\$2.101,44. Para a enzalutamida, o custo total de visitas médicas foi de R\$ 860,16 (Tabela 6). Os EAs de AA+P custaram $R \$ 1.912,62$, enquanto para enzalutamida esse resultado foi de $R \$ 1.786,07$. Desta maneira, o custo total de tratamento durante o período de 12 meses foi mais elevado para AA+P, com R\$ 130.246,02, quando comparado à enzalutamida, com $\mathrm{R} \$ 127.198,79$.

Por meio do NNT obtido e do custo total de tratamento anual, foi possível fazer o cálculo de COPE. Considerando o desfecho clínico de SG, o COPE de enzalutamida resultou em R\$ 1.626.582,99, enquanto para AA+P esse valor foi de $\mathrm{R} \$ 15.144 .885,88$, promovendo uma diferença anual de R\$ 13.518.302,89, por evento prevenido. A SLPr demonstrou uma diferença anual de R\$ 284.666,64 entre enzalutamida (R\$ 329.701,37) e AA+P (R\$ 614.368,01), favorecendo o tratamento com enzalutamida (Figura 1 e Tabela 7).

\section{Horizonte de tempo 24 meses}

Os dados clínicos foram obtidos durante o tempo de 24 meses, utilizando a curva atualizada de ambos medicamentos para os dois desfechos estudados (SG e SLPr). A porcentagem de pacientes vivos em 24 meses foi de 0,72 de enzalutamida versus 0,63 do placebo, enquanto para $\mathrm{AA}+\mathrm{P}$ foi de 0,69 ver- 
Tabela 1. Preço e custo dos medicamentos utilizados

\begin{tabular}{lcccccc}
\hline Medicamento & Preço CMED & Custo unitário & Unidades por mês & Custo em 12 meses & Custo em 24 meses \\
\hline Enzalutamida & $R \$ 10.377,92$ & $R \$ 86,48$ & 120 & $R \$ 124.535,04$ & $R \$ 175.635,92$ \\
\hline Abiraterona & $R \$ 10.482,82$ & $R \$ 87,36$ & 120 & $R \$ 125.793,84$ & $R \$ 166.047,87$ \\
\hline Prednisona & $R \$ 12,17$ & $R \$ 0,61$ & 60 & $R \$ 438,12$ & $R \$ 578,32$ \\
\hline AA+P & - & - & - & $R \$ 126.232,96$ & $R \$ 166.626,19$ \\
\hline
\end{tabular}

${ }^{*} \mathrm{AA}+\mathrm{P}$ : acetato de abiraterona + prednisona

Tabela 2. Dose e frequência de administração de acetato de abiraterona e enzalutamida

\begin{tabular}{ll}
\hline Medicamento & Posologia \\
\hline Enzalutamida & 4 comprimidos de $40 \mathrm{mg}$ por dia \\
\hline Acetato de abiraterona & 4 comprimidos de $250 \mathrm{mg}$ por dia \\
\hline Prednisona & 2 comprimidos de $5 \mathrm{mg}$ por dia \\
\hline
\end{tabular}

Tabela 3. Custos por evento adverso (graus 3 e 4, com porcentagem $>=1 \%$, e casos de eventos adversos especiais) (Beer et al., 2014; U.S. Food and Drug Administration, 2016a, 2016b; Ryan et al., 2015)

\begin{tabular}{|c|c|c|c|}
\hline Evento adverso & Custo por $\mathrm{EA}^{*}$ & ENZ & $\mathrm{AA}+\mathrm{P}$ \\
\hline Falha aguda renal & $R \$ 8.544,69$ & $1,0 \%$ & $0,0 \%$ \\
\hline $\begin{array}{l}\text { Insuficiência } \\
\text { adrenocortical }\end{array}$ & $R \$ 9.050,02$ & $0,0 \%$ & $0,5 \%$ \\
\hline Astenia/fadiga & $\mathrm{R} \$ 645,18$ & $3,4 \%$ & $2,2 \%$ \\
\hline Fibrilação atrial & $\mathrm{R} \$ 4.947,37$ & $0,0 \%$ & $0,0 \%$ \\
\hline Dor nas costas & $\mathrm{R} \$ 170,94$ & $2,5 \%$ & $0,0 \%$ \\
\hline Eventos cardíacos & $\mathrm{R} \$ 25.852,78$ & $3,0 \%$ & $6,0 \%$ \\
\hline Dispnéia & $\mathrm{R} \$ 1.099,01$ & $0,6 \%$ & $2,4 \%$ \\
\hline Edema & $\mathrm{R} \$ 188,08$ & $0,2 \%$ & $0,4 \%$ \\
\hline Queda & $\mathrm{R} \$ 4.024,50$ & $1,6 \%$ & $0,0 \%$ \\
\hline Hematúria & $\mathrm{R} \$ 209,31$ & $1,3 \%$ & $1,3 \%$ \\
\hline Hipertensão & $R \$ 2.960,75$ & $7,2 \%$ & $3,9 \%$ \\
\hline Inchaço na articulação & $R \$ 192,15$ & $1,0 \%$ & $2,0 \%$ \\
\hline $\begin{array}{l}\text { Infecção do trato } \\
\text { respiratório inferior }\end{array}$ & $\mathrm{R} \$ 6.349,55$ & $1,5 \%$ & $0,0 \%$ \\
\hline Fratura não patológica & $R \$ 24.051,17$ & $2,1 \%$ & $0,0 \%$ \\
\hline $\begin{array}{l}\text { Síndrome de encefalopatia } \\
\text { posterior reversível }\end{array}$ & $\mathrm{R} \$ 10.713,15$ & $0,1 \%$ & $0,0 \%$ \\
\hline Convulsão & $\mathrm{R} \$ 1.778,54$ & $0,1 \%$ & $0,0 \%$ \\
\hline Aumento do ALT & $R \$ 402,35$ & $0,2 \%$ & $6,1 \%$ \\
\hline Aumento do AST & $\mathrm{R} \$ 402,35$ & $0,0 \%$ & $3,1 \%$ \\
\hline Hiperglicemia & $R \$ 227,10$ & $0,0 \%$ & $6,5 \%$ \\
\hline Hipocalemia & $R \$ 580,92$ & $0,0 \%$ & $2,8 \%$ \\
\hline Linfopenia & $R \$ 974,74$ & $0,0 \%$ & $8,7 \%$ \\
\hline
\end{tabular}

AA: acetado de abiraterona + prednisona; EA: evento adverso; ENZ: enzalutamida;

*Micro-custeio baseado na tabela Classificação Brasileira

Hierarquizada de Procedimentos Médicos (CBHPM).
Tabela 4. Dados clínicos (sobrevida global e sobrevida livre de progressão) em 12 meses

\begin{tabular}{lccl}
\hline & ENZ & Placebo & Referência \\
\hline SG & $92 \%$ & $84 \%$ & PREVAiL (Beer et al., 2015) \\
\hline SLPr & $66 \%$ & $28 \%$ & PREVAiL (Beer et al., 2015) \\
\hline & AA+P & P+P & Referência \\
\hline SG & $91 \%$ & $90 \%$ & COU-AA-302 (Ryan et al., 2015) \\
\hline SLPr & $58 \%$ & $37 \%$ & COU-AA-302 (Ryan et al., 2013) \\
\hline
\end{tabular}

AA+P: acetato de abiraterona + prednisona; ENZ: enzalutamida;

P+P: placebo + prednisona; SG: sobrevida global; SLPr:

sobrevida livre de progressão radiográfica

Tabela 5. Número necessário a tratar (NNT) em 12 meses

\begin{tabular}{lcccc}
\hline & ENZ & IC95\% & AA+P & IC95\% \\
\hline NNT (SG) & 12,79 & $8,80-23,38$ & 116,28 & $27-$ Inf \\
\hline NNT (SLPr) & 2,59 & $2,36-2,87$ & 4,72 & $4,10-5,55$ \\
\hline
\end{tabular}

AA+P: acetato de abiraterona + prednisona; ENZ: enzalutamida; IC: intervalo de confiança; NNT: número necessário a tratar; SG: sobreviga global; SLPr: sobrevida livre de progressão radiográfica.

Tabela 6. Custos de acompanhamento de paciente em 12 meses

\begin{tabular}{lccc}
\hline & Enzalutamida & AA+P* & Custo unitário† \\
\hline Visitas médicas & 12 & 12 & $\mathrm{R} \$ \mathbf{7 1 , 6 8}$ \\
\hline Função hepática & - & 16 & $\mathrm{R} \$ \mathbf{7 7 , 5 8}$ \\
\hline Total & $\mathbf{R} \$ \mathbf{8 6 0 , 1 6}$ & $\mathbf{R} \$ \mathbf{2 . 1 0 1 , 4 4}$ & - \\
\hline
\end{tabular}

*AA+P: acetato de abiraterona + prednisona;

tDados obtidos da tabela Classificação Brasileira Hierarquizada de Procedimentos Médicos (CBHPM).

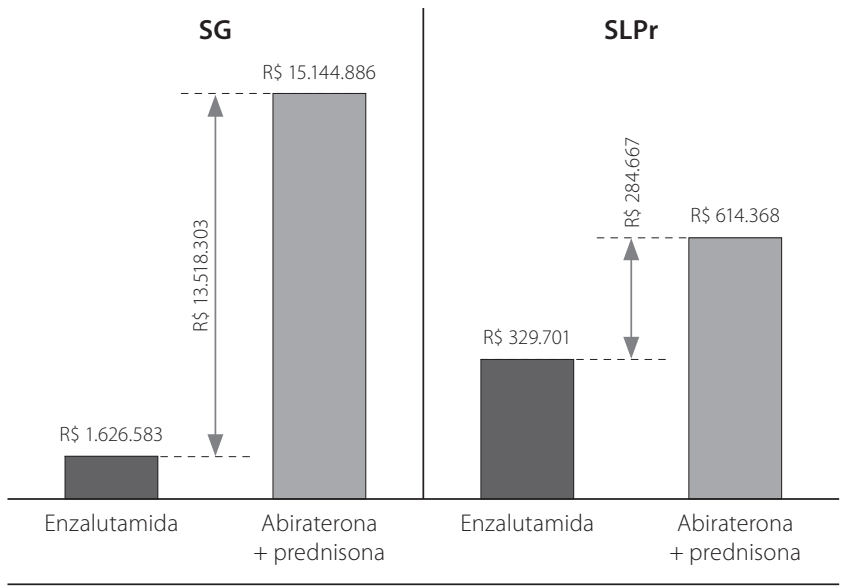

Figura 1. Resultados de COPE em 12 meses 
Tabela 7. Custo por evento evitado (COPE) em 12 meses

\begin{tabular}{lccccc}
\hline & ENZ & IC95\% & AA+P & IC95\% & Diferença Anual \\
\hline COPE (SG) & $R \$ 1.626 .582,99$ & $(1.119 .367-2.974 .335)$ & $R \$ 15.144 .885,88$ & $(3.688 .176-$ Inf $)$ & $-R \$ 13.518 .302,89$ \\
\hline COPE (SLPr) & $R \$ 329.701,37$ & $(300.179-365.664)$ & $R \$ 614.368,01$ & $(534.222-722.806)$ & $-R \$ 284.666,64$ \\
\hline
\end{tabular}

AA+P: acetato de abiraterona + prednisona; ENZ: enzalutamida; COPE: Custo por evento evitado; SG: sobreviga global; SLPr: sobrevida livre de progressão radiográfica

sus 0,63 do braço de placebo associado à prednisona. A SLPr foi de 0,45 para enzalutamida versus 0,18 do placebo, já para $A A+P$, os resultados foram de 0,38 para $A A+P$ versus 0,18 de placebo+prednisona (Tabela 8).

O NNT está exemplificado na Tabela 9. A enzalutamida apresentou menores valores de NNT considerando ambos os desfechos clínicos, SG e SLPr.

Para o horizonte de tempo de 24 meses, assim como mencionado anteriormente, a duração de tratamento foi calculada por meio da AUC da curva de SLPr de cada um dos medicamentos, assumindo que o paciente interrompe o tratamento no momento de progressão da doença. A enzalutamida apresentou um tempo de tratamento maior, de 16,9 meses, enquanto a AA+P apresentou uma duração de tratamento de 15,8 meses.

O custo de acompanhamento de AA+P no período de 24 meses resultou na necessidade de avaliar 19 exames hepáticos e aproximadamente 16 visitas médicas, resultando em R\$ $2.609,43$. Enquanto o tratamento completo de enzalutamida necessitou de aproximadamente 17 visitas médicas, totalizando no valor de R\$1.213,11 (Tabela 10). Os EAs avaliados foram os mesmos durante o período de 12 meses. Desta maneira, o custo total de tratamento durante o período de 24 meses foi de R\$178.659,81 para enzalutamida e R\$ 171.148,24

Tabela 8. Dados clínicos (sobrevida global e sobrevida livre de progressão) em 24 meses

\begin{tabular}{lccl}
\hline & ENZ & Placebo & Referência \\
\hline SG & $72 \%$ & $63 \%$ & PREVAiL (Beer et al., 2015) \\
\hline SLPr & $45 \%$ & $18 \%$ & PREVAiL (Beer et al., 2015) \\
\hline & AA+P & P+P & Referência \\
\hline SG & $69 \%$ & $63 \%$ & COU-AA-302 (Ryan et al., 2015) \\
\hline SLPr & $38 \%$ & $18 \%$ & COU-AA-302 (Ryan et al., 2013) \\
\hline
\end{tabular}

AA+P: acetato de abiraterona + prednisona; ENZ: enzalutamida; P+P: placebo + prednisona; SG: sobrevida global; SLPr: sobrevida livre de progressão radiográfica.

Tabela 9. Número necessário a tratar (NNT) em 24 meses

\begin{tabular}{lcccc}
\hline & ENZ & IC95\% & AA+P & IC95\% \\
\hline NNT (SG) & 11,0 & $7,34-21,98$ & 16,56 & $10,34-41,48$ \\
\hline NNT (SLPr) & 3,58 & $3,22-4,03$ & 5,00 & $4,49-5,64$ \\
\hline
\end{tabular}

AA+P: acetato de abiraterona + prednisona; ENZ: enzalutamida; IC: intervalo de confiança; NNT: Número necessário a tratar; SG: sobreviga global; SLPr: sobrevida livre de progressão radiográfica. para AA+P. O valor superior obtido com o tratamento com enzalutamida deveu-se ao maior tempo de utilização desse medicamento.

O COPE de enzalutamida resultou em $\mathrm{R} \$ 1.965 .454$, enquanto a AA+P em $R \$ 2.833 .580,08$, promovendo uma diferença de $R \$ 868.125,61$ por evento prevenido para o desfecho de SG. A SLPr demonstrou uma diferença de R\$216.414,61 entre enzalutamida (R\$ 639.326,58) e AA+P (R\$ 855.741,18), favorecendo o tratamento com enzalutamida (Figura 2 e Tabela 11).

\section{Discussão}

O estudo sugere enzalutamida como uma alternativa de tratamento mais eficiente, pois é uma opção terapêutica clinicamente efetiva, concomitantemente a um valor de investimento menor ao ser comparado com o tratamento com acetato de abiraterona. Essa afirmação é consequência dos resultados obtidos de NNT e COPE.

Tabela 10. Custo de acompanhamento de paciente em 24 meses

\begin{tabular}{lccc}
\hline & Enzalutamida & AA+P* & Custo unitário† \\
\hline Visitas médicas & 17 & 19 & $\mathrm{R} \$ \mathbf{7 1 , 6 8}$ \\
\hline Função hepática & - & 16 & $\mathrm{R} \$ \mathbf{7 7 , 5 8}$ \\
\hline Total & R\$ 1.213,11 & R\$ 2.609,43 & - \\
\hline
\end{tabular}

*AA+P: acetato de abiraterona + prednisona;

+Dados obtidos da tabela Classificação Brasileira

Hierarquizada de Procedimentos Médicos (CBHPM).

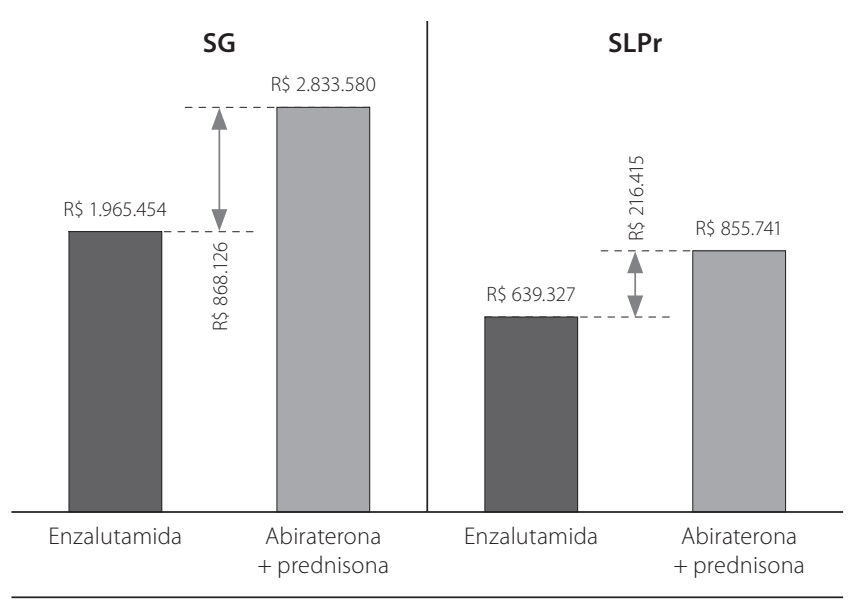

Figura 2. Resultados de COPE em 24 meses 
Tabela 11. Custo por evento evitado (COPE) em 24 meses

\begin{tabular}{lccccc}
\hline & ENZ & IC95\% & AA+P & IC95\% & Diferença Anual \\
\hline COPE (SG) & $R \$ 1.965 .454,47$ & $(1.310 .665-3.927 .653)$ & $R \$ 2.833 .580,08$ & $(1.769 .989-7.099 .975)$ & $-R \$ 868.125,61$ \\
\hline COPE (SLPr) & $R \$ 639.326,58$ & $(575.138-719.643)$ & $R \$ 855.741,18$ & $(768.622-965.134)$ & $-R \$ 216.414,61$ \\
\hline
\end{tabular}

AA+P: acetato de abiraterona + prednisona; ENZ: enzalutamida; COPE: Custo por evento evitado; SG: sobreviga global; SLPr: sobrevida livre de progressão radiográfica.

O NNT e COPE para ambas opções terapêuticas, tanto no horizonte de tempo de 12 meses e de 24 meses, apresentaram resultados menores para enzalutamida em comparação à combinação de AA+P. Quanto menor o valor de NNT, menor a necessidade de número de pacientes para se observar um desfecho de interesse. Da mesma maneira, quanto menor o valor de COPE, menor o custo de se evitar um evento indesejado. Portanto, por meio desses dados é possível discutir que os resultados de enzalutamida foram mais favoráveis tanto em aspectos econômicos quanto clínicos.

Essa análise está de acordo com a metodologia do estudo de 2015 de Stefani e colaboradores (Stefani et al., 2015), com algumas diferenças, principalmente relacionadas ao cálculo de custo de tratamento total. Além do custo de aquisição de medicamentos, adicionou-se os custos de acompanhamento e custos de manejo de EAs ao custo de tratamento total. Além disso, outra diferença nos cálculos foi a aplicação da porcentagem de $4 \%$ nos pacientes sendo tratados com enzalutamida, para representar os indivíduos que utilizariam a prednisona concomitantemente ao medicamento, como descrito no estudo PREVAIL. Por meio dessas diferenças metodológicas, alguns problemas com possíveis vieses apontados na análise de 2015 foram minimizados.

Entretanto, a principal diferença do atual estudo e da análise realizada em 2015 foi a adição de um horizonte de tempo mais longo. A utilização de dois horizontes de tempo (12 e 24 meses) foi justificada pela necessidade de utilizar os dados clínicos de um ponto da curva mais afastada em relação ao tempo de 12 meses. Ao analisar as curvas de SG do estudo COU-AA-302 (Ryan et al., 2015), se observou que no ponto de 12 meses não havia diferença significativa entre as curvas de $\mathrm{AA}+\mathrm{P}$ e placebo associado à prednisona, o que poderia tornar a análise enviesada e sensível a pequenas variações de SG, e consequentemente, impactar no resultado de NNT. Por esse motivo, optou-se por um horizonte temporal de longo prazo onde foi possível observar uma separação mais adequada entre as curvas do estudo clínico.

Vale ressaltar que algumas limitações ainda foram observadas durante o desenvolvimento do estudo. Dentre elas, deve-se citar a utilização de estudos distintos de cada medicamento devido à falta de estudos de comparação direta entre enzalutamida e abiraterona. Essa comparação pode promover dúvidas sobre a precisão dos resultados, considerando que os dois ensaios clínicos são originalmente diferentes. Ainda, deve-se destacar que não é possível concluir a influência dos diferentes braços controles entre os estudos PREVAIL e COU-AA-302, com a adição de prednisona ao grupo placebo nos resultados do estudo de abiraterona (Beer et al., 2015; Ryan et al., 2015).

Adicionalmente, há o componente da extrapolação dos dados clínicos para o mundo real. Os dados clínicos refletem resultados referentes a uma população de pacientes em meio controlado, que não refletem necessariamente uma população de pacientes sem monitoramento clínico constante.

Apesar dessas limitações, este estudo possibilitou realizar uma avaliação mais simplificada com utilização de dados clínicos e econômicos disponíveis atualmente. Essa análise poderia se tornar uma ferramenta auxiliar na tomada de decisão, mesmo na ausência de avaliações de custo-efetividade ou de comparações diretas da eficácia das duas drogas.

\section{Conclusão}

Os resultados de NNT e COPE, independentemente do desfecho estudado (SG ou SLPr), foram menores para a enzalutamida quando comparada a abiraterona em combinação com prednisona em ambos os horizontes de tempo estudados de 12 e 24 meses.

\section{Referências bibliográficas}

Associação Médica Brasileira. Classificação Brasileira Hierarquizada de Procedimentos Médicos. São Paulo: AMB; 2015. 204 p.

Astellas Farma Brasil Importação e Distribuição de medicamento Ltda. Xtandi® (enzalutamida) [Bula]. 2016.

Beneficência Portuguesa de São Paulo; Sociedade Beneficente Israelita Brasileira [site na Internet]. Manual de Oncologia Clínica no Brasil [atualizado 2016 jan 30; citado 2016 maio 14]. Disponível em: https:// mocbrasil.com/

Beer T,.Armstrong AJ, Sternberg CN, et al. Enzalutamide (ENZA) in men with chemotherapy-Naïve metastatic castration-resistant prostate cancer (mCRPC): Final analysis of the phase 3 PREVAlL study. J Clin Oncol. 2015;33:5036.

Beer TM, Armstrong AJ, Rathkopf DE et al. Enzalutamide in metastatic prostate cancer before chemotherapy. N Engl J Med. 2014;371(5):424-33.

Hotte SJ, Saad F. Current management of castrate-resistant prostate cancer. Current Oncology. 2010;17(Suppl 2):S72-S79.

Janssen-Cilag Farmacêutica Ltda. Zytigatm (acetato de abiraterona) [Bula]. 2016

Maharaj, R. Adding cost to NNT: the COPE statistics. ACP Journal Club. 2008;148, (1): pA8 
Ministério da Saúde (Brasil).Agência Nacional de Vigilância Sanitária(ANVISA). Orientações gerais para a notificação de eventos adversos relacionados à assistência à saúde. Brasília: Ministério da Saúde; 2015.27 p.

Ministério da Saúde (Brasil). Agência Nacional de Vigilância Sanitária (ANVISA). [site na Internet]. Câmara de Regulação do Mercado de Medicamentos (CMED). [atualizado 2016 jan 30; citado 2016 maio 14]. Disponível em: <http://portal.anvisa.gov.br/wps/ portal/anvisa/anvisa/posuso/regulacaodemercado/!ut/p/c4/04_ SB8K8xLLM9MSSzPy8xBz9CP0os3hnd0cPE3MfAwN_Dz8DA09_c19vrw AXA4MAM_2CbEdFAGk6puA!/?1dmy\&urile=wcm:path:/anvisa+portal/ anvisa/pos+-+comercializacao+--pos+-+uso/regul>.

Ministério da Saúde (Brasil). Instituto Nacional do Câncer José Alencar Gomes da Silva (INCA). [site na Internet]. Atlas On-line de Mortalidade. [atualizado 2016 jan 30; citado 2016 maio 14]. Disponível em: https:// mortalidade.inca.gov.br/MortalidadeWeb/

Ministério da Saúde (Brasil). Instituto Nacional do Câncer José Alencar Gomes da Silva (INCA). Estimativa 2016 - Incidência de câncer no Brasil. Brasília: Ministério da Saúde; 2015. 127 p.

National Comprehensive Cancer Network (NCCN). NCCN Guideline - Prostate cancer. 2016.

National Cancer Institute. [site na Internet]. Surveillance, Epidemiology, and End Result Program. [atualizado 2015 set 24; citado 2016 maio 14]. Disponível em: http://seer.cancer.gov/

Parker C, Gillessen S, Heidenreich A et al. Cancer of the prostate: ESMO Clinical Practice Guidelines for diagnosis, treatment and follow-up. Ann Oncol. 2015; 26 (suppl. 5): v69-v77.
Recine F.; Sternberg CN. Hormonal therapy and chemotherapy in hormonenaive and castration resistant prostate cancer. Transl Androl Urol. 2015;4(3):355-64.

Ryan CJ, Smith MR, Fizazi K, et al. Abiraterone acetate plus prednisone versus placebo plus prednisone in chemotherapy-naive men with metastatic castration-resistant prostate cancer (COU-AA-302): final overall survival analysis of a randomised, double-blind, placebo-controlled phase 3 study. Lancet Oncol. 2015;16(2):152-60.

Ryan CJ, Smith MR, de Bono JS et al. Abiraterone in metastatic prostate cancer without previous chemotherapy. N Engl J Med. 2013 Jan 10;368(2):138-48.

Stefani, S. et al. Custo por evento evitado da enzalutamida versus abiraterona associada à prednisona em homens com câncer de próstata resistente à castração metastático, sob a perspectiva do Sistema de Saúde Suplementar no Brasil. Jornal Brasileiro de Economia da Saúde. 2015; 7(3): 127.

U.S. Food and Drug Administration. Xtandi ${ }^{\oplus}$ (enzalutamide) [Bula]. 2016a.

U.S. Food and Drug Administration. ZytigaTM (abiraterone acetate) [Bula]. 2016b.

World Health Organization (WHO) [site na Internet]. Globocan 2012: Estimated cancer incidence, mortality and prevalence worldwide in 2012. [atualizado 2015 set 24; citado 2016 maio 14]. Disponível em: http:// globocan.iarc.fr/Default.aspx 